\title{
Bulk density estimation using a 3-dimensional image acquisition and analysis system
}

\author{
Adam Heyduk ${ }^{1, a}$ \\ ${ }^{1}$ Silesian University of Technology, Department of Electrical Engineering and Control in Mining, 44-100 Gliwice, \\ ul. Akademicka 2, Poland
}

\begin{abstract}
The paper presents a concept of dynamic bulk density estimation of a particulate matter stream using a 3-d image analysis system and a conveyor belt scale. A method of image acquisition should be adjusted to the type of scale. The paper presents some laboratory results of static bulk density measurements using the MS Kinect time-of-flight camera and OpenCV/Matlab software. Measurements were made for several different size classes.
\end{abstract}

\section{Importance and range of bulk density measurements}

Bulk density, also called an apparent density, is defined as the mass per unit volume of loosely piled granular material (eg. coal or coke). The coal bulk density depending on its particle size, impurities, moisture may be in the range of 700 to $1100 \mathrm{~kg} / \mathrm{m}^{3}$. The bulk density varies depending on the way in which this material is stored. Under gravity load or under external pressure, spaces between the particles can be reduced, so the bulk density may increase over time. The bulk density, like the material volume, is particularly important for aggregates used in civil engineering $[1,2]$ since they act as volume fillers to concrete mixes. However, this parameter is important also for coal [3], especially in the case of measuring the stored coal amount in the landfill. This was sanctioned in national regulation, according to which "the mass of stored coal is determined by weight or calculated as the product of volume and bulk density." This is connected with coal excise taxation and with trading $\mathrm{CO}_{2}$ emission rights. However it should be noticed that, in accordance with [3] the regulation method of calculating the weight of the stored coal on the basis of the volume and bulk density, is only valid for coarse size classes. Therfore, the analysis performed in this paper was restricted to the coarse size classes.

\section{Static and dynamic measurements of bulk density}

National standards and regulations define the density measurement in a static manner [3] by using the box the with size dependent on the coal particle size (the larger the particle size the greater the box dimensions). The mass of the coal sample is simply defined as a difference of the full box mass and empty box mass. This rule of a discrete measurement is presented demonstratively in Fig. 1. However,

${ }^{\text {a }}$ Corresponding author: Adam.Heyduk@polsl.pl 
this process needs sampling from the greater volume, so only the small part of the total amount can be measured.

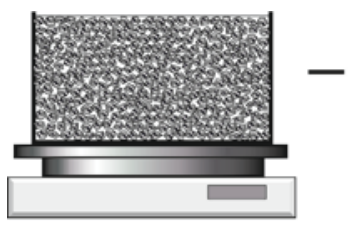

mass

of the full box

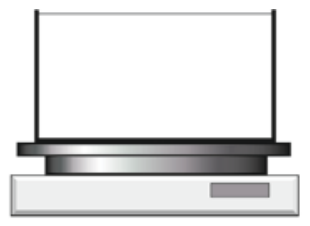

mass

of empty box

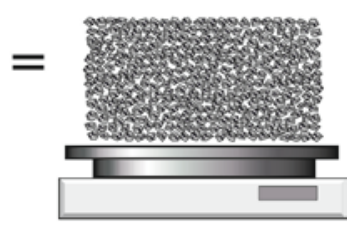

mass

of coal or

other mineral

particles

Figure 1. Schematic of discrete (static) bulk density measurement.

It would be desirable to measure bulk density in a continuous way, as it makes possible to measure the whole stream of the granular material. However, this method needs continuous and synchronous measurements of both mass and volume of the whole stream. The result of mass measurement should be divided by the corresponding result of the volume measurement. Mass measurements can be performed by widely used belt conveyor scale, and volume measurement can be performed by recently developed 3-dimensional image acquisition and analysis. There are several methods of 3dimensional image acquisition. The most applicable in these case are laser triangulation [4] and timeof flight cameras [5-7]. Each of these methods has its own advantages and disadvantages in terms of cost, efficiency and precision. However, the most important difference are the time and space range of these methods. The laser triangulation measures simultaneously the profile of a single line and the time-of-flight camera measures usually a rectangular area (as it has a matrix generator and converter). So, the selection of the method should adjusted to the type of the conveyor belt scale used in a particular application. If there is a single sensor scale measuring only one line, a laser triangulation system [4] presented in Fig. 2, should be used. However, if there is a multi-sensor scale measuring the mass of the greater load area, a time-of-flight camera capturing larger field of vision $[5,6]$ presented in Fig. 3, should be used.

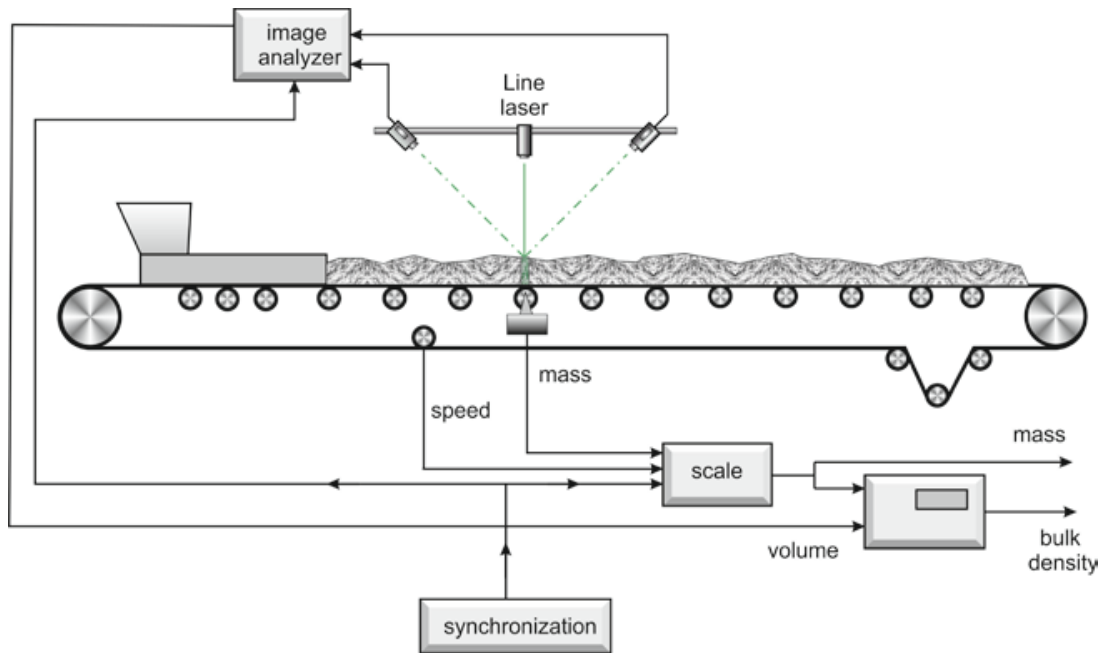

Figure 2. Continuous measurement of bulk density using a single-sensor scale and laser triangulation. 


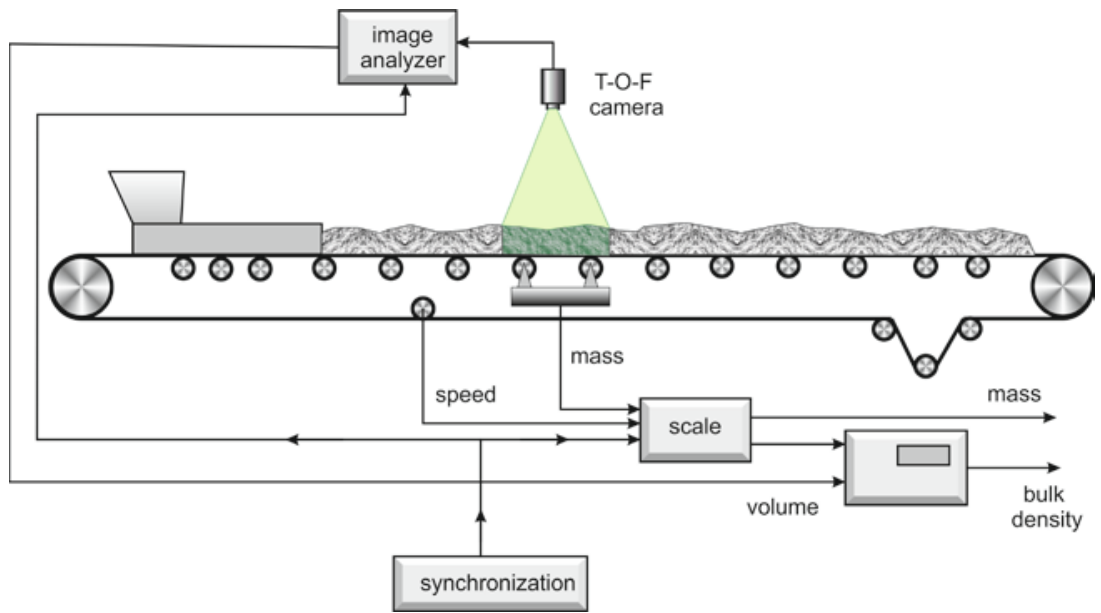

Figure 3. Continuous measurement of bulk density using a multi-sensor scale and time-of-flight measurement camera.

\section{Volume estimation methods}

Volume estimation algorithms are much simpler then algorithms for calculation of the particle size distribution, because they do not require precise delineation of single touching and overlapping particles (this is usually the most complex, error-prone time-consuming task [4]). There is also no need to analyse the lower, usually invisible from the top, hemisphere of individual particle, because the lower surface of the whole particle population is well known (it is just the upper surface of the conveyor belt). The most important part of the volume estimation algorithm is to calculate a height difference between the surface of the particle stream and surface of an empty conveyor belt, adopted as a reference surface. So, the height map $h_{d}(x, y)$ of the particulate material stream is:

$$
h_{d}(x, y)=h_{s}(x, y)-h_{b}(x, y)
$$

where $h_{s}(x, y)$ is depth map of the stream surface and $h_{b}(x, y)$ is depth map of the empty conveyor belt. If for any point $P(x, y)$ the belt area is locally empty:

$$
h_{s}(x, y)=h_{b}(x, y)
$$

then

$$
h_{d}(x, y)=0
$$

and this point $P$ will not be considered in any further volume calculations. Each pixel $P(x, y)$ (where $x \in X, y \in Y$ ) of the 3 -d image represents in a horizontal plane a distinct patch with a constant elementary area $A_{e}$. The value of $A_{e}$ is dependent on image resolution and camera field of view angle. In the 3-d space it can be considered as a vertical stack of voxels (elementary volume elements), as presented in Fig. 4. Therefore, the volume of the mineral particle stream can be calculated as the sum of these voxel vertical stacks:

$$
V=\sum_{X} \sum_{Y} h_{d}(x, y) \cdot A_{e}=A_{e} \sum_{x \in X} \sum_{y \in Y} h_{d}(x, y) .
$$




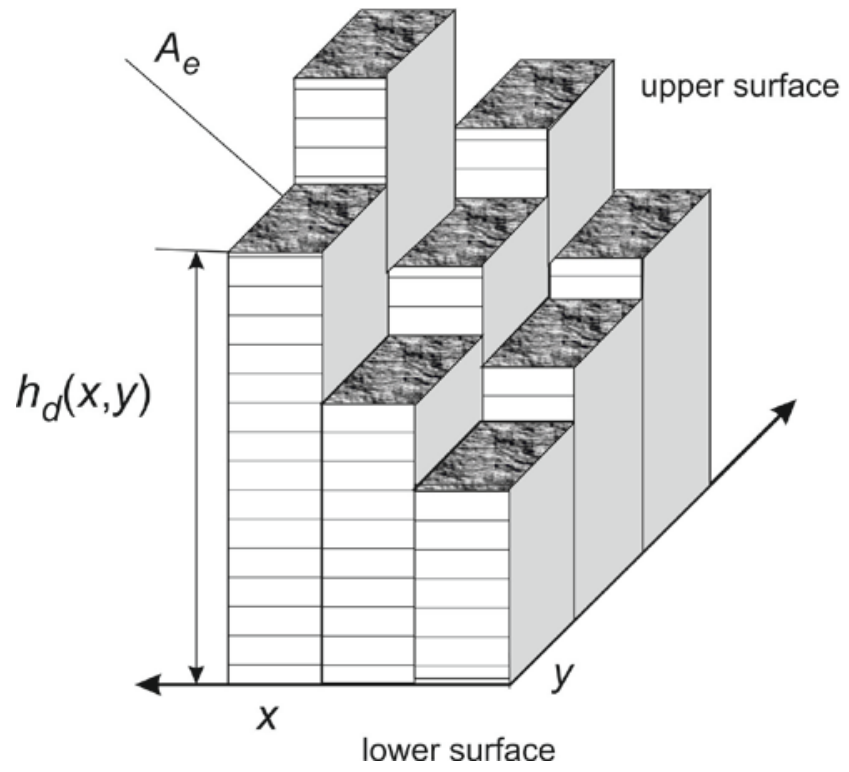

Figure 4. Outline of volume estimation based on the 3-d image analysis according to Eq. (4).

\section{Results of laboratory research}

Preliminary studies were performed is a laboratory equipped with a section of the conveyor belt, and the MS Kinect v2 time-of-flight camera with vertical precision $1 \mathrm{~mm}$ and horizontal resolution of $512 \times 424$ pix [7]. The pixel elementary area value $A_{e}$ was calculated as $0.85 \mathrm{~mm}^{2} /$ pix. It was estimated on the basis of image analysis performed for a flat rectangular reference object with known dimensions. The coal sample mass was measured using the digital scale with $0.001 \mathrm{~kg}$ resolution. The experiments were performed for different size classes presented in Table 1.

Table 1. Measurement and calculated results for coal particle size classes.

\begin{tabular}{|c|c|c|c|}
\hline $\begin{array}{c}\text { Size class } \\
{[\mathbf{m m}]}\end{array}$ & Mass $[\mathbf{k g}]$ & $\begin{array}{c}\text { Volume } \\
{\left[\mathbf{m}^{\mathbf{3}}\right]}\end{array}$ & $\begin{array}{c}\text { Bulk density } \\
{\left[\mathbf{k g} / \mathbf{m}^{\mathbf{3}}\right]}\end{array}$ \\
\hline $80-160$ & 7.076 & 0.0106 & 724 \\
\hline $50-80$ & 2.936 & 0.0038 & 777 \\
\hline $40-50$ & 3.656 & 0.0041 & 888 \\
\hline $30-40$ & 1.905 & 0.0020 & 963 \\
\hline $20-30$ & 3.936 & 0.0040 & 972 \\
\hline $10-20$ & 4.536 & 0.0045 & 1.010 \\
\hline $0-10$ & 4.638 & 0.0045 & 1.021 \\
\hline
\end{tabular}

The results of bulk density estimation from Table 1 are presented graphically in Fig. 5. The smaller the size class. The higher its bulk density. 


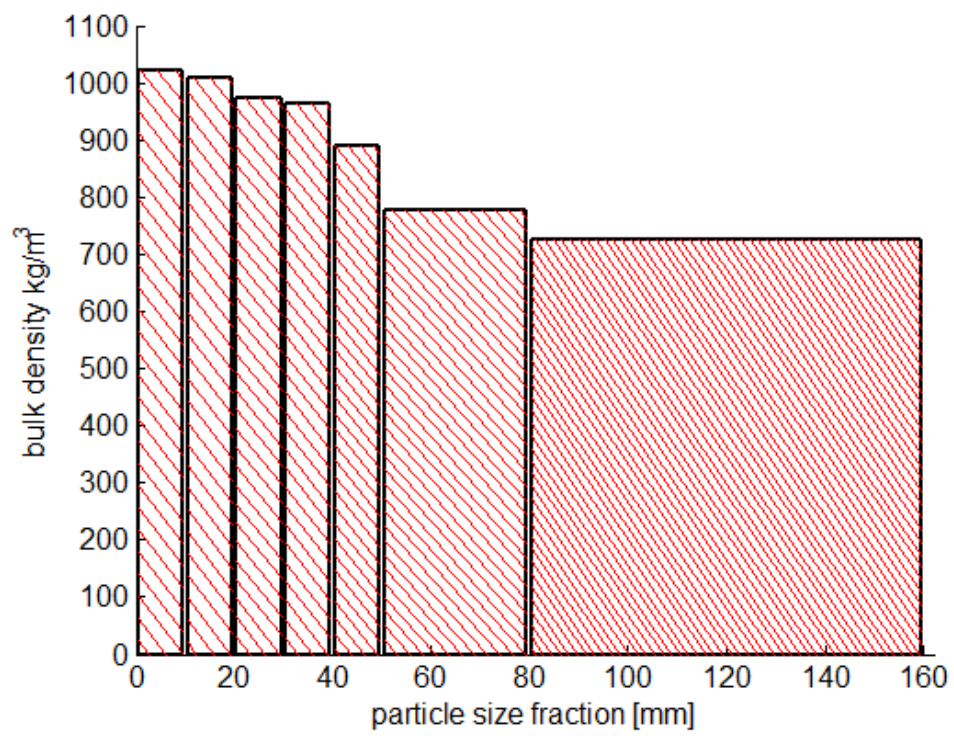

Figure 5. Results of bulk density estimation for different size classes from Table 1.

For comparison purposes, some different acquired (raw) and processed (differential without conveyor belt, ready for volume calculation) depth maps: one set for coarse particles population (size class $80-120 \mathrm{~mm})$, one set for medium particles $(30-40 \mathrm{~mm})$ and one for the finest particles e $(0-10$ $\mathrm{mm})$ were presented. In each case the reference depth map of an empty conveyor belt presented in Fig. 6 was used. The map for coarse particles on the conveyor belt was presented in Fig. 7. After subtracting the image of the empty conveyor belt (Fig. 6) we can get an image of the particles alone (Fig. 8). It is ready for volume calculation according to Eq. (4). Similar images for a medium size class $(30-40 \mathrm{~mm})$ were presented respectively in Figs. 9 and 10 and for the finest size class $(0-10$ $\mathrm{mm}$ ) in Figs. 11 and 12. All dimensions on these figures were presented in $\mathrm{mm}$.

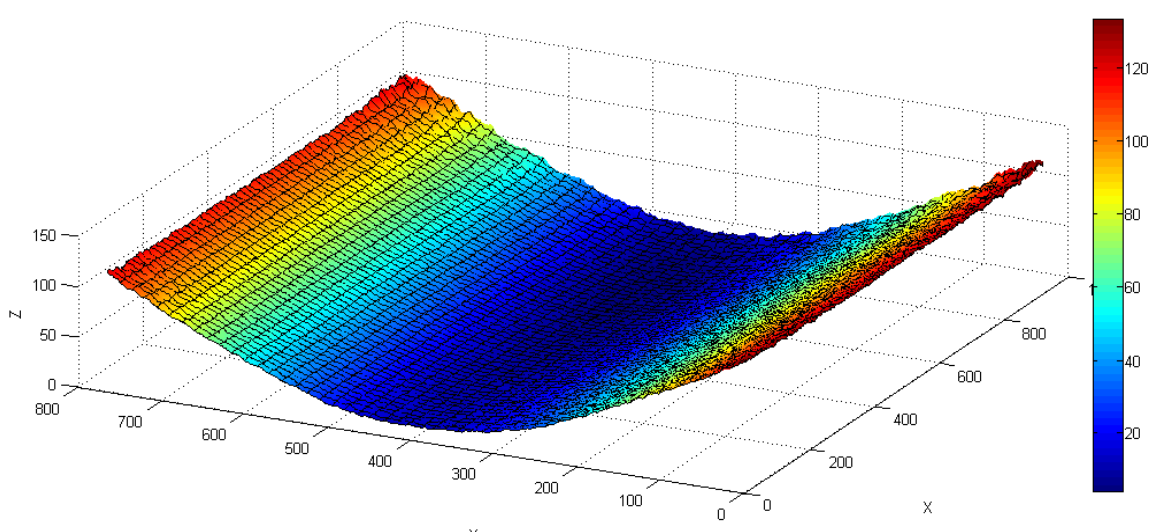

Figure 6. Depth map of an reference empty conveyor belt. 


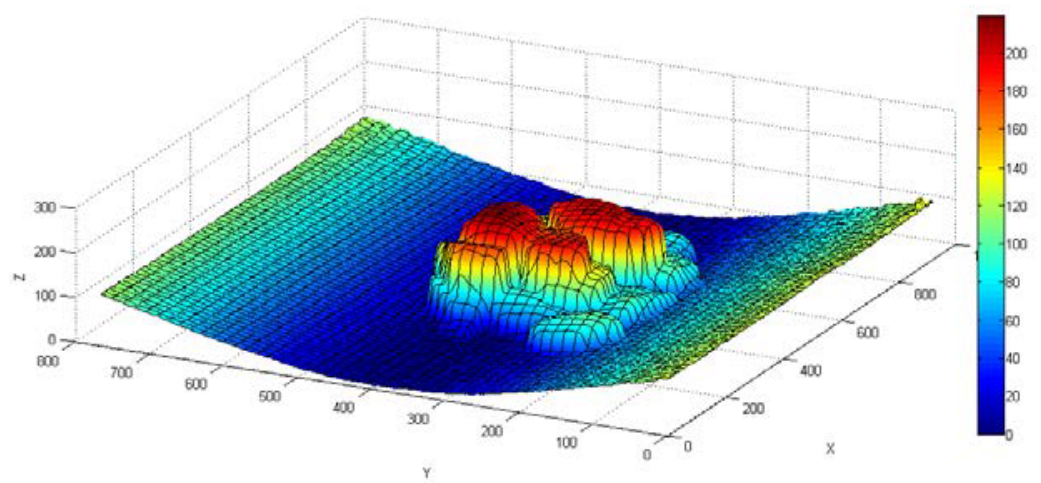

Figure 7. Raw depth map of coarse $(80-160 \mathrm{~mm})$ coal particles on the conveyor belt.

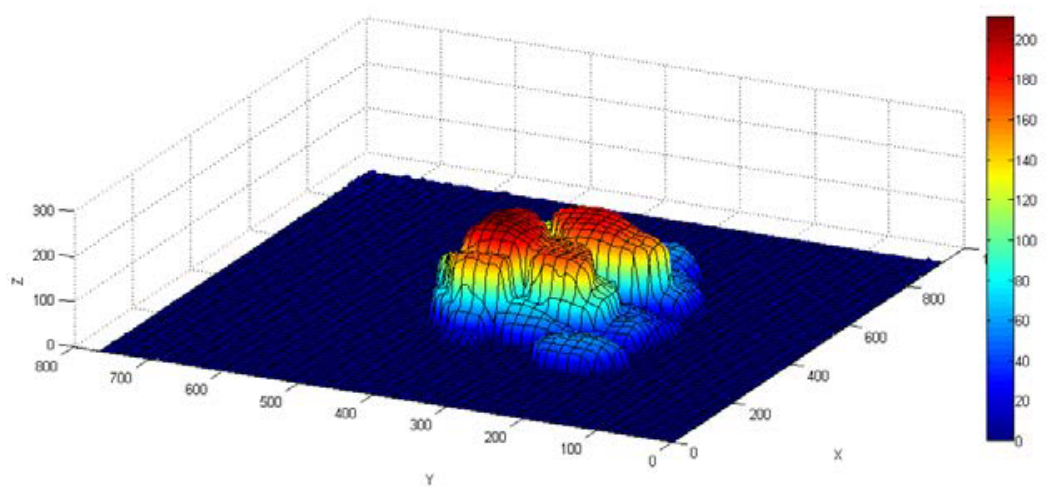

Figure 8. Differential depth map of coarse $(80-160 \mathrm{~mm})$ coal particles alone (without conveyor belt), ready for volume calculation.

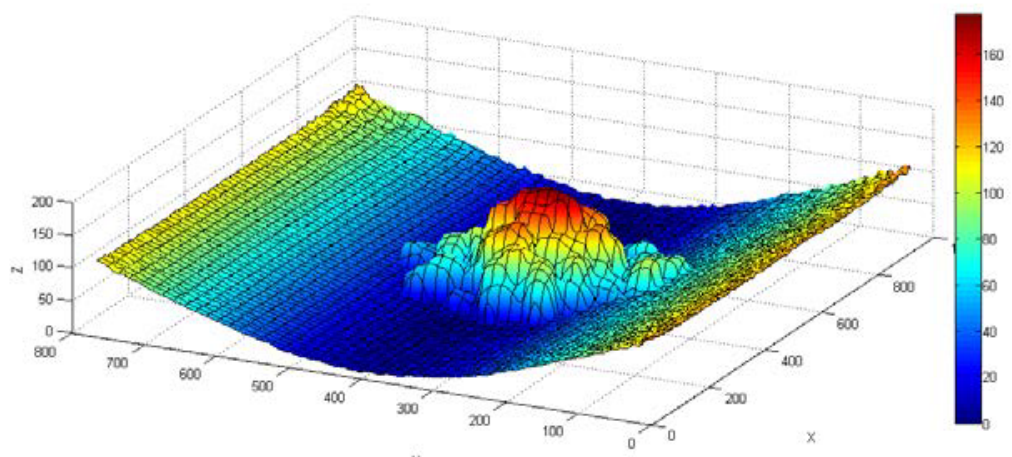

Figure 9. Raw depth map of medium (30 - $40 \mathrm{~mm}$ ) coal particles on the conveyor belt. 


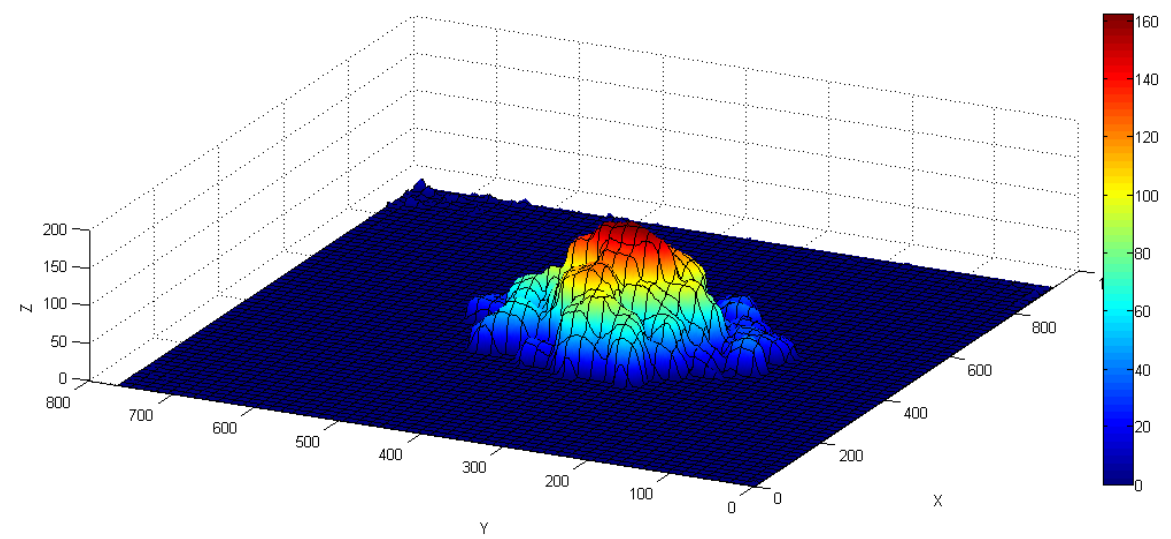

Figure 10. Differential depth map of medium $(30-40 \mathrm{~mm})$ coal particles alone (without conveyor belt), ready for volume calculation.

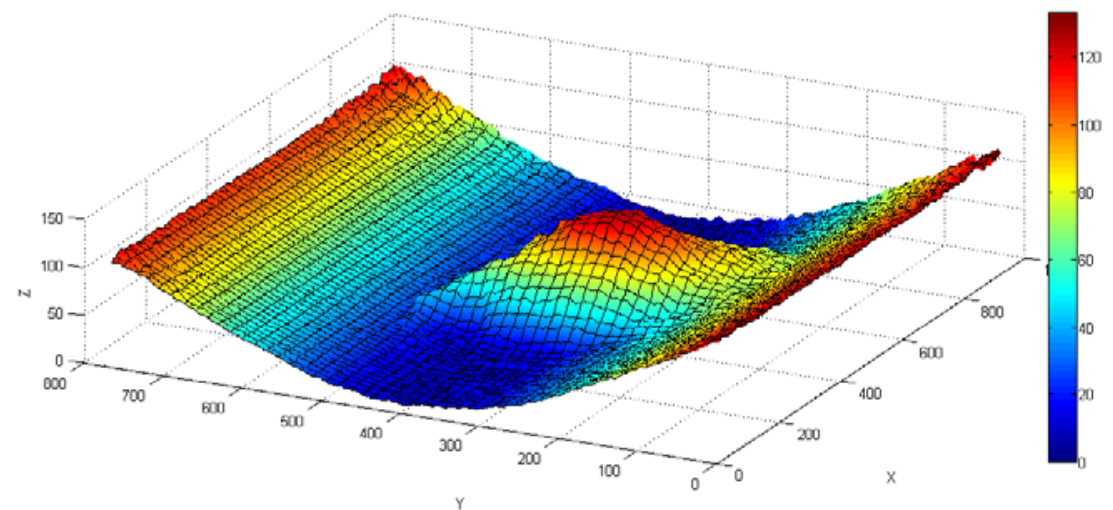

Figure 11. Raw depth map of fine $(0 \ldots 10 \mathrm{~mm})$ particles on the conveyor belt.

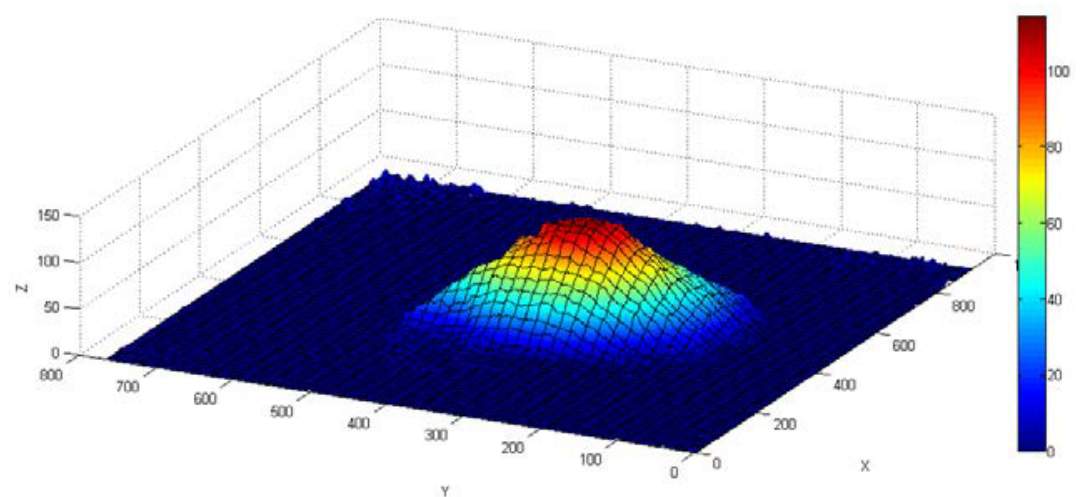

Figure 12. Differential depth map of fine $(0 \ldots 10 \mathrm{~mm})$ coal particles alone (without conveyor belt), ready for volume calculation. 


\section{Conclusions}

Modern 3-d image acquisition methods enable continuous measurement of bulk density for mineral particle streams. It can be useful for estimation of the stored bulk material amount e.g in a stockpile. Measurements can be made just on the conveyor belt, using belt the scale and image acquisition system. Volume estimation algorithms are relatively simple, as they do not require precise delineation of individual particles. There must be created a differential image. It can be obtained by subtracting the image of the empty conveyor belt from the image of the particle stream on the belt. The 3-d images, also called either depth maps or height maps, can be obtained by either laser triangulation or time-of-flight measurements. As the bulk density is connected to the particle size, it can be also used, under special conditions, as the rough indicator of the average particle size in a stream.

\section{References}

1. H. Zhang, Building materials in civil engineering (Science Press, Beijing, 2011)

2. W.F. Chen, J.Y. Liew, The Civil Engineering Handbook (CRC Press, Boca Raton, 2002)

3. L. Róg, R. Rompalski. Eng. Res. Report of CMI, (2012).

4. A. Heyduk, Arch. Min. Sci., 61, 15 (2016).

5. M. Hansard, S. Lee, O. Choi, R. Horaud. Time-of-Flight Cameras, Principles, Methods and Applications (Springer, London, 2013)

6. F. Remondino, D. Stoppa, TOF Range-Imaging Cameras (Springer, Heidelberg, 2013)

7. L.Yang, L. Zhang, H. Dong, IEEE Sens. J., 15, 42 (2015) 